\title{
ARTICLE
}

\section{A vacuole-like compartment concentrates a disordered calcium phase in a key coccolithophorid alga}

\author{
Sanja Sviben ${ }^{1, \star}$, Assaf Gal ${ }^{1,2, \star}$, Matthew A. Hood ${ }^{1,2,}$, Luca Bertinetti ${ }^{2}$, Yael Politi ${ }^{2}$, Mathieu Bennet ${ }^{2}$, \\ Praveen Krishnamoorthy ${ }^{1}$, Andreas Schertel ${ }^{3}$, Richard Wirth ${ }^{4}$, Andrea Sorrentino ${ }^{5}$, Eva Pereiro ${ }^{5}$, \\ Damien Faivre $^{2} \&$ André Scheffel $^{1}$
}

Coccoliths are calcitic particles produced inside the cells of unicellular marine algae known as coccolithophores. They are abundant components of sea-floor carbonates, and the stoichiometry of calcium to other elements in fossil coccoliths is widely used to infer past environmental conditions. Here we study cryo-preserved cells of the dominant coccolithophore Emiliania huxleyi using state-of-the-art nanoscale imaging and spectroscopy. We identify a compartment, distinct from the coccolith-producing compartment, filled with high concentrations of a disordered form of calcium. Co-localized with calcium are high concentrations of phosphorus and minor concentrations of other cations. The amounts of calcium stored in this reservoir seem to be dynamic and at a certain stage the compartment is in direct contact with the coccolith-producing vesicle, suggesting an active role in coccolith formation. Our findings provide insights into calcium accumulation in this important calcifying organism.

\footnotetext{
${ }^{1}$ Max-Planck Institute of Molecular Plant Physiology, Potsdam-Golm 14476, Germany. ${ }^{2}$ Department of Biomaterials, Max-Planck Institute of Colloids and Interfaces, Potsdam-Golm 14476, Germany. ${ }^{3}$ Carl Zeiss Microscopy GmbH, Global Applications Support, Oberkochen 73447, Germany.

${ }^{4}$ Department of Geomaterials, GeoForschungsZentrum Potsdam, Potsdam 14473, Germany. ${ }^{5}$ ALBA Synchrotron Light Source, Cerdanyola del Vallés, Barcelona 08290, Spain. ${ }^{*}$ These authors contributed equally to this work. Correspondence and requests for materials should be addressed to A.S. (email: Scheffel@mpimp-golm.mpg.de).
} 
C occolithophorid algae, a key phytoplankton group in the oceans, form elaborate arrays of minute calcite crystals known as coccoliths using $\mathrm{Ca}^{2+}$ and $\mathrm{HCO}_{3}^{-}$from the surrounding seawater ${ }^{1}$. This biomineralization process is of immense importance for the global cycle of carbon and other elements, as coccolithophore calcification sequesters massive deposits of $\mathrm{CaCO}_{3}$ into sea-floor sediments ${ }^{2,3}$. Coccolith deposits are widely used to infer past environmental conditions as environmental traces become incorporated into coccolith calcite during its formation ${ }^{4,5}$. Despite the widespread geoscientific importance of coccolithophore calcification, the cellular pathways that supply coccolith formation with the 'building blocks' and control the elemental and isotopic composition of the final mineral remain unidentified. Uncovering these pathways will not only provide a mechanistic framework for interpreting compositional data but also the necessary foundation to address why and how coccolith calcification will be affected by the projected ocean acidification and how this process will adapt to new environmental conditions.

In the bloom-forming species Emiliania huxleyi, which is the best studied and dominant coccolithophore in modern oceans, coccolith formation proceeds inside an intracellular membrane system termed as 'coccolith vesicle-reticular body' . Coccoliths are formed one at a time, as fast as one per hour, and require for an average coccolith, $22.5 \mathrm{fmol}$ of $\mathrm{Ca}^{2+}$ to be transferred into the coccolith vesicle 7 . The prevailing notion for coccolith calcite formation is that ions are transported through the cell to the site of calcite formation ${ }^{8,9}$. However, the classical, water-based preparation protocols used so far ${ }^{10-12}$ undermined the possibility of identifying highly soluble, amorphous Ca phases ${ }^{13}$ that, alternatively to ions, may be transported to the mineralization site as was discovered for other biomineralforming organisms ${ }^{14}$.

Here we investigated E. huxleyi cells spectroscopically and microscopically using preparation techniques that preserve soluble, amorphous Ca phases. Using a combination of cryosoft X-ray tomography and spectroscopy, and cryo-focused ion beam scanning electron microscopy (FIB-SEM), we visualized and characterized a highly concentrated, previously unidentified, pool of intracellular calcium and studied its corresponding ultrastructural environment. We show, using elemental analysis and live-cell staining, that polyphosphates and other elements, among them the paleomarker element $\mathrm{Mg}^{5}$, are co-localized with calcium, and present data that point to a possible route how calcium and other elements could be transferred to the site of mineralization.

\section{Results}

Speciation of intracellular calcium during coccolith formation. Our initial investigation of E. huxleyi for possible amorphous precursor phases of coccolith calcite involved X-ray absorption near-edge structure (XANES) spectroscopy. Cryogenic XANES is uniquely suited to discriminate calcium species in mixtures and played a pivotal role in the discovery of soluble inorganic phases during the formation of crystalline biominerals ${ }^{15,16}$. To follow intracellular Ca during the deposition of fresh coccolith calcite, we raised 'calcite-free' E. huxleyi cells (Supplementary Fig. 1), induced calcite formation by adding $\mathrm{Ca}^{2+}$ to the medium and cryo-preserved cells at 10 -min intervals up to $30 \mathrm{~min}$, which is when calcite crystals of status nascendi coccoliths are detectable by cross-polarized light microscopy (Supplementary Fig. 1b). The XANES spectrum of cells before induction $(0 \mathrm{~min})$ had a small shoulder at 4,060 eV, which increased over time and is indicative for calcite formation (Fig. 1a). We fitted the spectra of the induced cells using linear combinations of several reference spectra (Fig. 1b). The differences between the different linear combinations were minor, even though the best fits were obtained when using amorphous calcium carbonate (ACC) in addition to $\mathrm{CaCl}_{2}$ solution and calcite (Fig. 1a; see Supplementary Fig. 2a,b for fits with other reference spectra). This suggests that yet unidentified amorphous Ca phases are a significant fraction of intracellular calcium at all time points (Fig. 1c).

X-ray imaging of calcium. To directly observe the putative amorphous Ca phases, we investigated cryo-preserved E. huxleyi cells using synchrotron soft X-ray tomography and spectromicroscopy. The minimal sample preparation procedure, consisting only of dissolving extracellular coccoliths and vitrification of cells after de novo coccolith formation had started, allowed the visualization of the internal organization of the cells during coccolith production in a yet unprecedented close-to-native state. Reconstruction of tilt-series images taken at an X-ray energy of $520 \mathrm{eV}$ yielded three-dimensional (3D) data with a calculated spatial resolution of $52 \mathrm{~nm}$, half pitch. At this energy, C-rich and Ca-rich moieties are more absorptive than aqueous solutions and therefore concentrated organic and inorganic matter appear dark, whereas the cytosol appears bright ${ }^{17,18}$. The tomograms revealed intracellular bodies with highly absorbing material, similar to the calcite of coccoliths, which were always spatially separated from the coccolith in statu nascendi (Fig. 2a). We probed the $\mathrm{Ca}$ content of these bodies by imaging cells at $342 \mathrm{eV}$, which is below the $\mathrm{Ca} \mathrm{L}_{2,3}$-edge where calcium is virtually transparent, and at $353.2 \mathrm{eV}$, which is above the $\mathrm{Ca}$ absorption edge ${ }^{19}$. The difference images of the two energies revealed a distinct $\mathrm{Ca}$-rich body in addition to a forming coccolith in the cells (Fig. 2b). To characterize the $\mathrm{Ca}$ phase of the bodies, we acquired spatially resolved $\mathrm{Ca}_{2,3}$-edge XANES spectra by sequentially imaging the same field of view and varying the energy across the $\mathrm{Ca} \mathrm{L}_{2,3}$-edge. Averaged spectra extracted from coccoliths in statu nascendi showed the characteristic spectrum of calcite (Fig. 2c). For reason explained below, we also acquired $\mathrm{Ca}_{2,3}$-edge XANES spectra from synthetic, poorly crystalline calcium phosphate (Fig. 2c). The averaged spectra extracted from the Ca-rich bodies lacked significant peaks at the energy of the calcite crystal field peaks (at 347.9 and $351.3 \mathrm{eV}$ ) or the hydroxyapatite crystal field peaks (at 348.1 and $351.5 \mathrm{eV}$ ), suggesting that the body contains an amorphous calcium phase $\mathrm{e}^{19-22}$. On the basis of the absorbance intensity at the Ca edge, which is proportional to the $\mathrm{Ca}$ content in the beam path and the volume of the Ca-rich body, the average calcium concentration in the body was determined to be $\sim 13.4 \pm 2.3 \mathrm{M}$.

3D ultrastructure of calcifying cells. To elucidate the ultrastructural environment of the Ca-rich body at higher resolution, we imaged cryo-fixed cells by serial FIB milling and block face SEM at cryo-condition using a Zeiss Auriga60 FIB-SEM ${ }^{23}$. In the energy selective backscattered (EsB) electron images, the Ca-rich bodies and coccoliths in statu nascendi were easily identifiable due to their calcite-like contrast (Fig. 3b,d). Image analysis, using coccolith calcite (calcium concentration is $27 \mathrm{M}$ ) and culture medium (calcium concentration is $10 \mathrm{mM}$ ) as $\mathrm{Ca}$ reference concentrations, yielded a concentration of $10 \mathrm{M} \mathrm{Ca}$ for the body, which is in range of the concentration determined from our X-ray tomography data set. In the in-lens secondary electron images, membrane-bound compartments were clearly visible (Fig. 3a,c). Most interestingly, a membrane was found in close proximity with the Ca-rich body, visible as thin dark line (Fig. 3c,e). Manual segmentation of this membrane in the image stacks revealed it to encompass the Ca-rich body, forming a compartment (Fig. 3f). Careful inspection of all images in the stacks found no connection between this compartment and the coccolith vesicle-reticular 
a

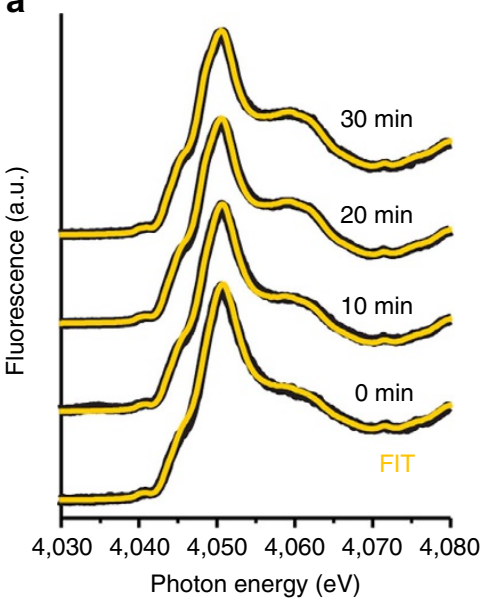

b

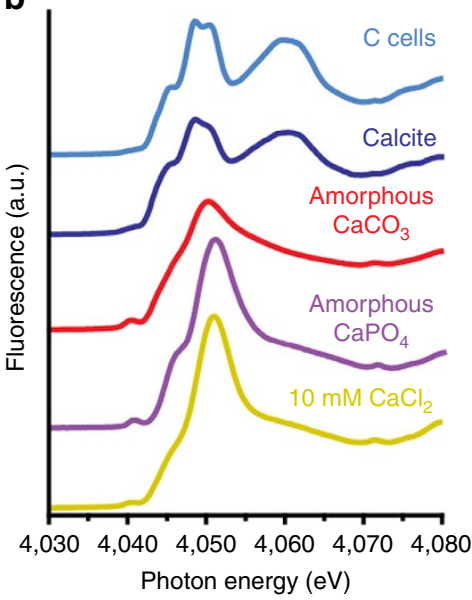

c

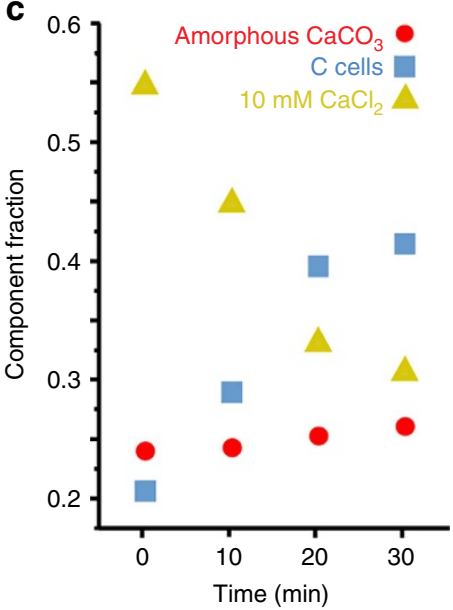

Figure 1 | Speciation of cellular calcium during the early stages of coccolith formation in $\mathbf{E}$. huxleyi. (a) Time-resolved evolution of the XANES spectra (black) of cells induced to form calcite and of the calculated fits (orange) using linear combinations of three reference standards (coccolith calcite, free calcium ions and amorphous $\mathrm{CaCO}_{3}$ ). (b) Ca K-edge XANES spectra of calcium reference standards and of $E$. huxleyi cells enclosed by a sphere of coccoliths ( $C$ cells). Free calcium ions were represented by $10 \mathrm{mM} \mathrm{CaCl}_{2}$ solution. The spectrum of amorphous calcium phosphate is courtesy of Diane Eichert, Elettra synchrotron, Trieste, Italy ${ }^{47}$. The spectrum of $\mathrm{C}$ cells showed the characteristic feature of calcite and was used instead of synthetic calcite for fitting the spectra of induced cells. (c) Relative contribution of the calcium references to the three component fits shown in a.
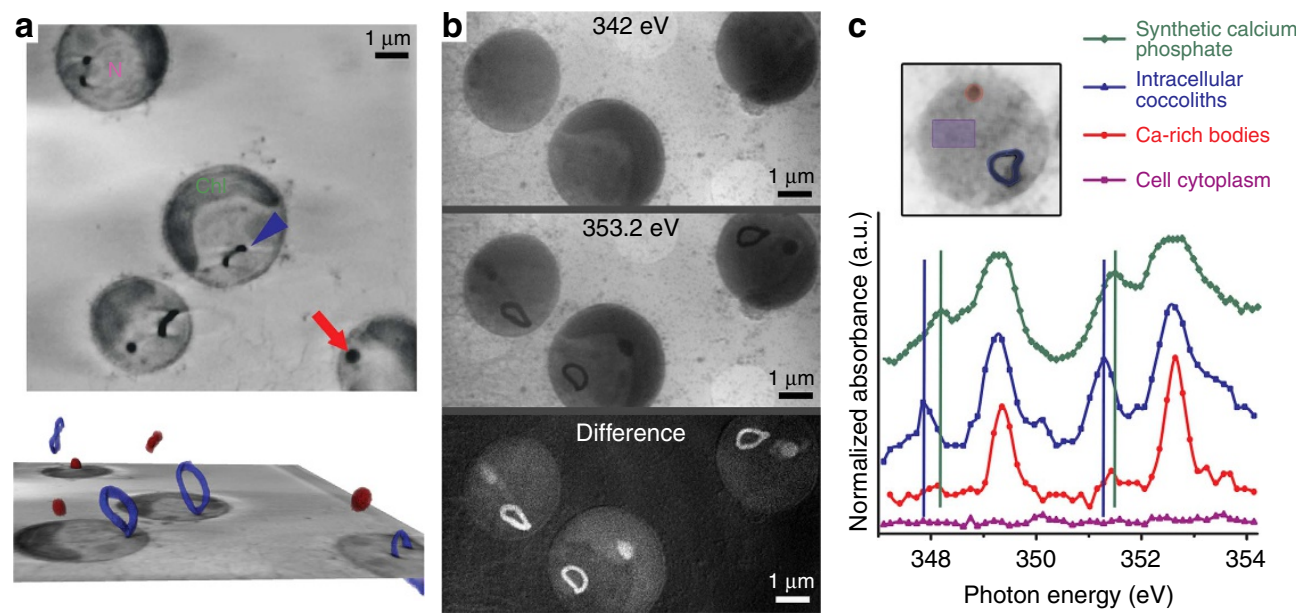

Figure 2 | Cryo-X-ray imaging reveals concentrated calcium pools in E. huxleyi cells. (a) Two-dimensional slices from a reconstructed X-ray tomogram with (top) an immature coccolith marked by the arrowhead and a calcium-rich body marked by the arrow, and (bottom) with 3D segmentation of the calcium-rich bodies (red) and intracellular coccoliths (blue). (b) X-ray images recorded at an energy below the Ca $\mathrm{L}_{2,3}$-egde ( $342 \mathrm{eV}$ ), at the edge energy $\left(353.2 \mathrm{eV}\right.$ ) and the grey value difference between both images. (c) Averaged XANES spectra of the $\mathrm{Ca}_{2,3}$-edge. For each spectrum, data from the relevant pixels of four cells were averaged; the inset shows the exact locations in one of these cells. Notice the difference in the position of the crystal field peak (vertical lines) between coccolith calcite and synthetic calcium phosphate. Figure is accompanied by Supplementary Movie 1.

body system or any other visible endomembrane system. Noteworthy, the compartment was always larger than the enclosed Ca-rich body and the largest compartment had a volume of $1.5 \mu \mathrm{m}^{3}$. If the largest compartment would be filled entirely with calcium at a concentration of $10 \mathrm{M}$, as in the Ca-rich body, it would store $15 \mathrm{fmol} \mathrm{Ca}$, which is about two-third of the $\mathrm{Ca}$ required for a full coccolith.

Compositional characterization of the Ca-rich body. We investigated the thin sections of high-pressure frozen, cryosubstituted and resin-embedded E. huxleyi cells using high-angle annular dark-field scanning transmission electron microscopy (HAADF-STEM) and analysed the elemental composition by energy-dispersive X-ray (EDX) spectroscopy and electron energy loss spectroscopy (EELS) at positions of interest. HAADF-STEM is suited for the imaging of the non-stained thin sections, as the image contrast is related to the atomic number of the elements in the beam path ${ }^{24}$. Calcium appears therefore brighter than biomolecules. We observed electron-dense Ca-rich bodies in the thin sections collected in ethylene glycol (Fig. 4a) but not in the sections collected in water. The dissolution of the bodies in water was accompanied by the dissolution of the coccolith calcite crystals, leaving an impression in the embedding resin. The same has been noticed previously by others ${ }^{12}$. The use of water for collecting the thin sections may therefore be the reason why the Ca-rich body was not observed in previous cytological studies in which occasionally a spacious intracellular compartment was observed but designated as chrysolaminarin vesicle ${ }^{10}$ or vacuole ${ }^{25}$ due to its highly electron transparent content, which most likely dissolved during sample preparation and could have been the Ca-rich body. When imaging the body at high magnification, 

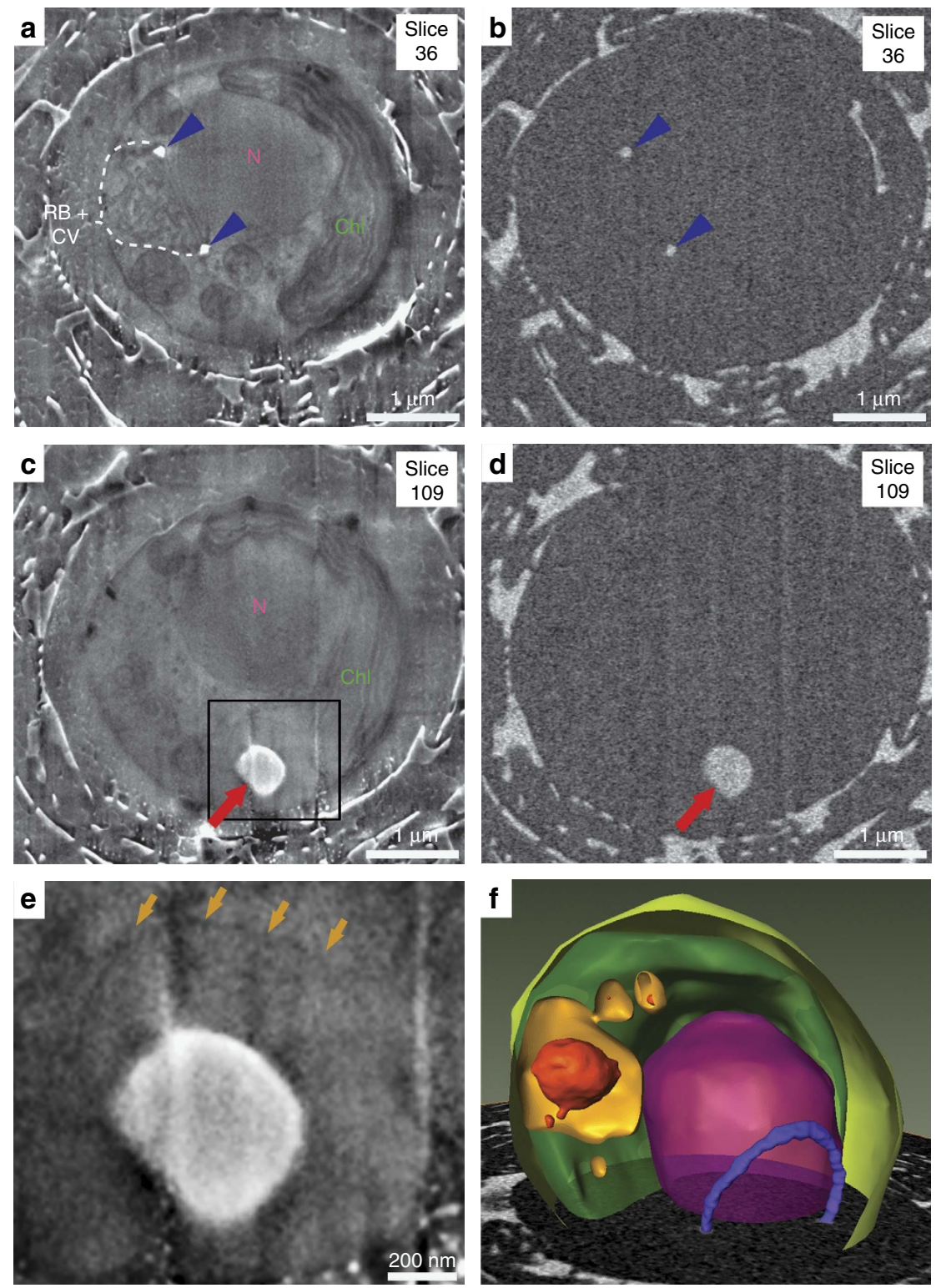

Figure 3 | Cryo-FIB-SEM imaging of vitrified E. huxleyi reveals coccolith calcite and calcium-rich bodies in separate compartments. (a-d) Two slices from the same cell acquired with in-lens secondary electron detector $(\mathbf{a}, \mathbf{c})$ and energy selective backscattered electron detector $(\mathbf{b}, \mathbf{c})$ showing a cross-sectioned coccolith in statu nascendi (blue), the Ca-rich body (red), the coccolith vesicle (CV)-reticular body system (RB), the nucleus (N) and the chloroplast (Chl). Additional organelles are visible in the secondary electron images as is shown in Supplementary Fig. 3. (e) Oversampled and contrastenhanced magnification of the area framed in c, illustrating the membrane that encloses the Ca-rich body (arrows). (f) 3D reconstruction of an E. huxleyi cell from a cryo-FIB-SEM image series, showing the nucleus (violet), chloroplast (dark green), plasma membrane (light green), a coccolith in statu nascendi (blue), Ca-rich bodies (red) and the membranes encompassing Ca-rich bodies (orange). Figure is accompanied by Supplementary Video 2.

no lattice fringes were observed, supporting our X-ray spectroscopy conclusion that the $\mathrm{Ca}$ in the body is in an amorphous phase (Fig. 4b). The carbon K-edge EELS spectra of the Ca-rich body was distinct from both coccolith calcite and the embedding resin, suggesting that the body does not contain significant amounts of calcium carbonate (Fig. 4c). Interestingly, the spectrum of the body showed a small peak at $288.2 \mathrm{eV}$, which has been previously associated with amide carbonyl bonds and suggests the presence of proteins ${ }^{26}$. To our surprise, the EDX analysis revealed the Ca-rich bodies to contain very high amounts of $\mathrm{P}$ in addition to $\mathrm{Ca}$ and minor amounts of other elements (Fig. 4d). Among these elements was magnesium, an important trace element in coccolith calcite, whose amount relative to calcium is used to infer past climatic conditions ${ }^{5}$. The excess of $\mathrm{P}$ over $\mathrm{Ca}$ in the Ca-rich body (Supplementary Table 1) suggests that $\mathrm{Ca}$ is bound by phosphate-rich macromolecules. Because the amount of $\mathrm{P}$ exceeds the amount of carbon, the most plausible candidates are not phosphorylated biomolecules but rather polyphosphates, which have been proposed to play a role in $\mathrm{CaCO}_{3}$ biomineralization ${ }^{27}$ and were discovered already in the non-calcifying relative of E. huxleyi, Pavlova ennorea sp. nov. ${ }^{28}$. In E. huxleyi polyphosphates may be synthesized by the enzyme polyphosphate polymerase (JGI ID406855) ${ }^{29}$.

We tested our polyphosphate hypothesis by live-cell confocal fluorescence microscopy using the fluorescent dye 4',6-diamidino-2-phenylindole (DAPI), which has been widely used to stain polyphosphates ${ }^{30}$, and the membrane-permeable calcium stain calcein-AM. The co-localized fluorescence signals from both the dyes in a compartment distinct from the nucleus and the coccolith vesicle-reticular body system (Fig. 5) are 

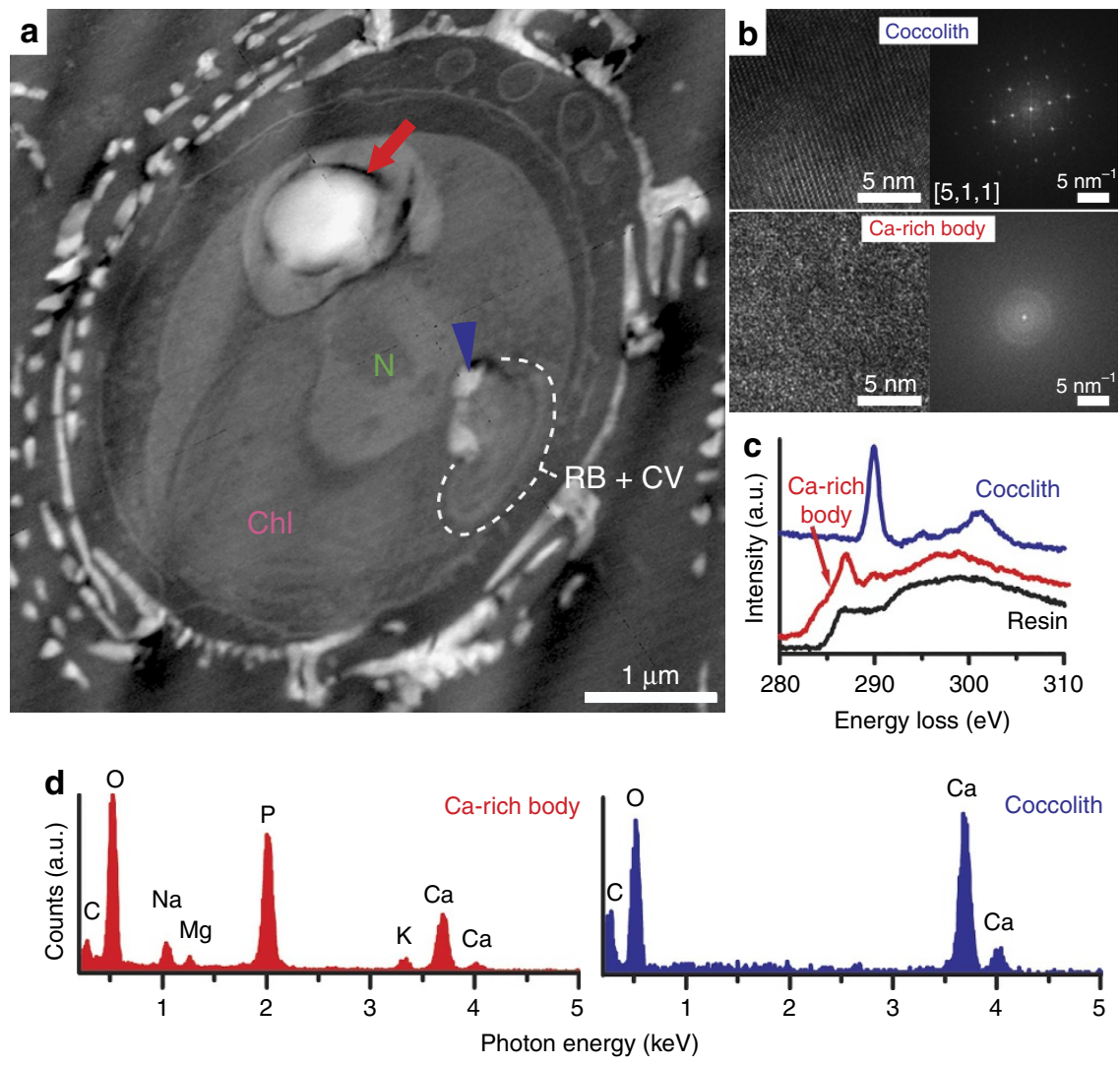

Figure 4 | Ultrastructural and elemental microanalysis of the calcium-rich body. (a) HAADF-STEM image of a thin-sectioned cell showing the nucleus $(\mathrm{N})$, the chloroplast $(\mathrm{Chl})$, the coccolith vesicle (CV)-reticular body system (RB) (encircled by the white line), coccolith calcite (blue arrowhead) and the Ca-rich body (red arrow). Additional organelles that are visible in the HAADF-STEM images are shown in Supplementary Fig. 4. (b) High-resolution images and corresponding Fourier-transformed image of coccolith calcite and the Ca-rich body. (c) STEM-EELS spectra measured at the carbon K-edge on the Ca-rich body, coccolith calcite and embedding resin. (d) STEM-EDX spectra of the Ca-rich body and coccolith calcite.

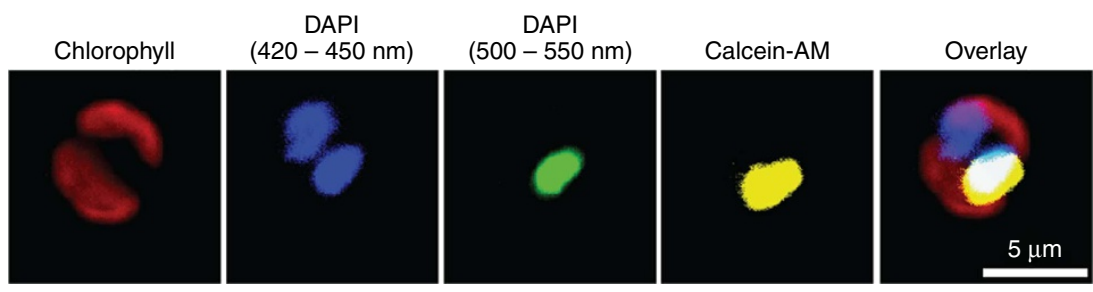

Figure 5 | Confocal fluorescence microscopy images of live $\boldsymbol{E}$. huxleyi. Cells were dual-stained with DAPI and the membrane-permeable calcium stain calcein-AM. The emitted fluorescence in the wavelength window between 420 and $450 \mathrm{~nm}$ originates from DAPI-DNA and DAPI-polyphosphate complexes, whereas the emission between 500 and $550 \mathrm{~nm}$ originates from DAPI bound to polyphosphate. The red channel shows the auto-fluorescence of chlorophyll.

consistent with our EDX results that strongly suggest that polyphosphate is complexing $\mathrm{Ca}$ in the Ca-rich compartment.

Variability of the Ca-rich body. The relatively low degree of synchronization between the cells of the same culture enabled the observation of variation in the appearance of the Ca-rich body. In the cryo-FIB-SEM images of some cells, a second phase with contrast intermediate between the cytoplasm and the Ca-rich body was observed, which is most likely also $\mathrm{Ca}$ but at a concentration lower than in the body (Fig. 6a-c). Assigning this second phase to be a more diluted, $\mathrm{Ca}$ pool is supported by a similar observation that was made in the cryo-soft-X-ray images, where some Ca-rich bodies were surrounded by a scattered cloud of low concentrations of calcium (Fig. 2a). Using coccolith calcite and culture medium as $\mathrm{Ca}$ reference concentrations, we calculated the average Ca concentration for several 'clouds' to be between 1 and $2 \mathrm{M}$. In accordance with these observations, we also saw in thin-sectioned cells a large compartment filled with diluted concentrations of $\mathrm{Ca}$ and $\mathrm{P}$ (Fig. 6d). The membranes delimiting the low concentrated $\mathrm{Ca}$ pools were seen in close contact with the coccolith vesicle-reticular body system (Fig. 6c,d). This configuration may allow the direct transfer of $\mathrm{Ca}$ and other trace elements found in coccolith calcite from the storage compartment into the coccolith vesicle.

\section{Discussion}

Our results show that E. huxleyi cells concentrate large amounts of $\mathrm{Ca}$ into a dense disordered phase in a vacuole-like compartment. Such a major internal store must be a dominant player in the cellular Ca budget and will have a pivotal role in the 

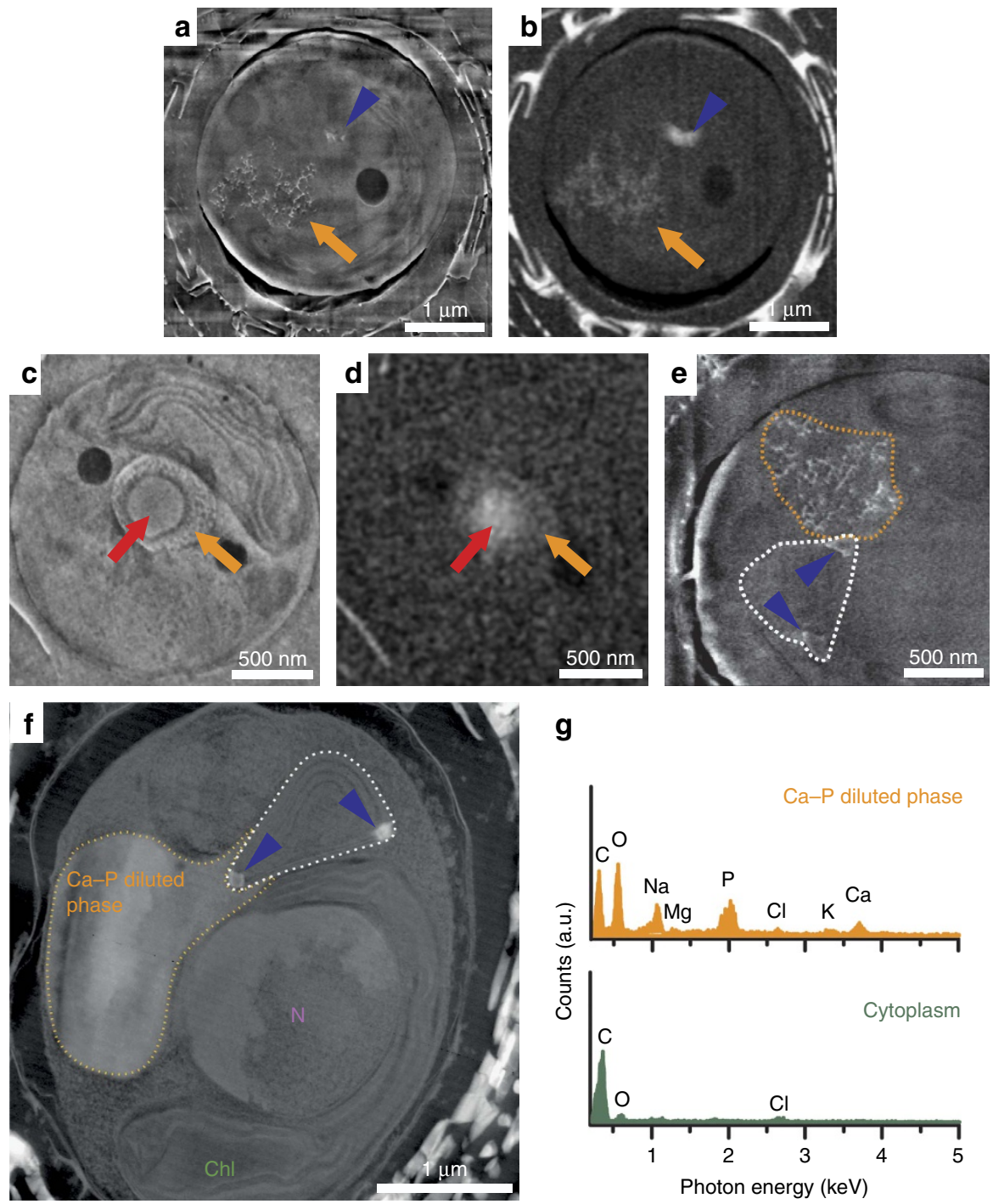

g

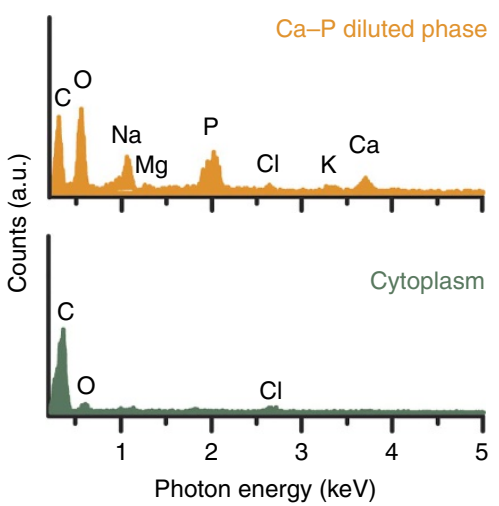

Figure 6 | Cells without Ca-rich body contained a compartment filled with diluted concentrations of calcium and phosphorus. (a-e) Slices from cryo-FIB-SEM image series of high-pressure frozen E. huxleyi cells imaged in secondary electron mode (a,c,e) and backscattered electron mode (b,d), showing coccolith calcite (blue arrowhead), the dense Ca-P-rich body (red arrow) and a pool of diluted concentrations of Ca (orange arrow, framed orange in $\mathbf{e}$ ) in close contact. The white line in $\mathbf{e}$ frames the coccolith vesicle-reticular body system. (f) HAADF-STEM image of a thin-sectioned cell showing the nucleus $(\mathrm{N})$, the chloroplast (Chl), coccolith calcite (blue arrowhead), the coccolith vesicle-reticular body system (framed white) and the vacuole-like compartment containing Ca and P (framed orange). (g) EDX spectra taken from inside the compartment framed orange in $\mathbf{f}$ and of cytosol. The epoxy resin contributes to the $\mathrm{C}$ peak and therefore the $\mathrm{C}$ signal does not represent in vivo concentrations.

subcellular partitioning and allocation of Ca. The Ca phase stored in the here-discovered compartment is likely not a direct precursor phase of coccolith calcite, as (i) the Ca-rich body was never seen in direct contact with status nascendi coccoliths, (ii) $\mathrm{Ca}$ and $\mathrm{P}$ were not detectable in the lumen of the coccolith vesicle-reticular body system and the coccolith calcite is virtually free of $\mathrm{P}$, and (iii) the calcein stain was never observed in the coccolith vesicle-reticular body system or coccolith calcite, ruling out the possibility of bulk transport from the Ca reservoir to the coccolith vesicle. The latter observation is in contrast to the situation in many other calcifying organisms where exogenously applied calcein becomes incorporated in the mineralized material ${ }^{14,31,32}$. Considering all the above-reported observations, the most plausible Ca transfer scenario is that $\mathrm{Ca}$ ions are released from their complex with polyphosphate, likely by enzymatic degradation of the polyphosphate and/or acidification of the compartment, and these are transported by $\mathrm{Ca}$ transporter and/or passive diffusion into the coccolith vesicle-reticular body system
(Fig. 7). The large membrane surface of the reticular body has been suggested to accommodate large numbers of calcium transporters ${ }^{33}$. The direct contact between the membranes of both compartment systems (Fig. 6c,d), and in particular between the $\mathrm{Ca}$ store and the reticular body, may be advantageous as it may enable direct transfer of $\mathrm{Ca}$ and so bypass the cytosol where a high concentration of calcium ions could be toxic.

$\mathrm{Ca}$-P-rich intracellular compartments have been reported also in multicellular $\mathrm{CaCO}_{3}$ mineralizing organisms ${ }^{34,35}$, but experimental challenges and the inherent complexity of these organisms have so far limited mechanistic investigation into their role in the mineral formation pathway. As simple unicellular organisms, E. huxleyi and other coccolithophores are attractive models for investigations on the cellular calcium budget and its use in $\mathrm{CaCO}_{3}$ biomineralization. The here-discovered component of the intracellular Ca pathway in E. huxleyi offers a new entry point into investigating the mechanistic details underlying coccolith formation and the incorporation of trace metals into 


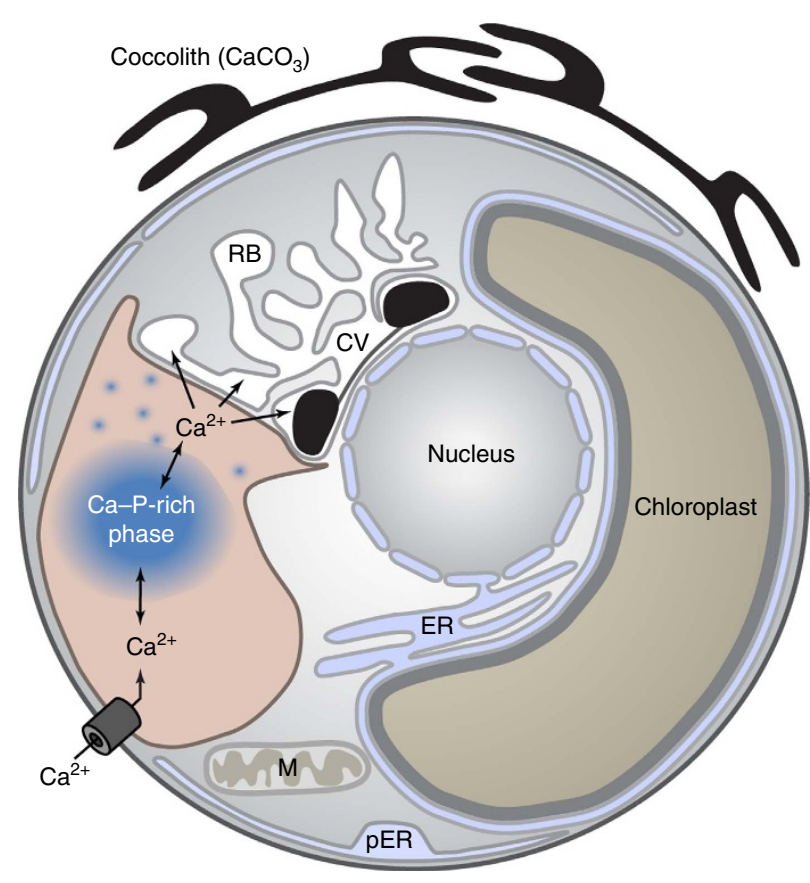

Figure 7 | Conceptual model of the coccolith calcium pathway.

$\mathrm{Ca}$ accumulation and calcite precipitation are spatially and temporally separated. Calcium uptake into cells involves $\mathrm{Ca}$ transporter. The $\mathrm{Ca}$ ions are concentrated by polyphosphates into a disordered phase in a compartment distinct from the coccolith vesicle-reticular body system. The disordered $\mathrm{Ca}$ phase is a dynamic reservoir, concentrating and dispatching $\mathrm{Ca}$ ions. The released $\mathrm{Ca}$ ions are possibly transferred into the coccolith vesicle-reticular body system by $\mathrm{Ca}$ transporter and/or passive diffusion driven by a high-concentration gradient in free $\mathrm{Ca}$ ions. Inside the coccolith vesicle the calcium is precipitated as calcite.

coccolith calcite. The challenge ahead is to decipher the biochemical and mineralogical mechanisms underlying the formation of the Ca-P-rich phase and the reorganization of this phase in relation to the formation of calcite. Interestingly, the here-described Ca-P-rich compartment shares several characteristics with acidocalcisomes of non-calcifying organisms ${ }^{36}$. This makes it tempting to speculate that both the compartments are evolutionary linked. Further studies are, however, required to address this hypothesis.

E. huxleyi is known to thrive in waters containing low amounts of $\mathrm{P}$, where other phytoplankton cannot survive ${ }^{39}$. The high $\mathrm{P}$ content of the Ca-rich body points to link between phosphate metabolism and calcification in E. huxleyi and may suggest that calcification is of functional significance for the ecological success of E. huxleyi.

Our findings for E. huxleyi raise the question how widespread this Ca pathway is in coccolithophores. Earlier studies revealed that in the species Pleurochrysis carterae, a significant amount of the calcium for synthesis of a coccolith is transported through the endomembrane system by acidic polysaccharide-Ca complexes termed coccolithosomes ${ }^{38}$. However, coccolithosomes have never been observed outside the genus Pleurochrysis, demonstrating that different $\mathrm{Ca}$ transport pathways evolved in coccolithophores.

The insights emerging from our study may bring the widespread interpretation of coccolith composition as proxy for seawater chemistry ${ }^{5}$ into a mechanistic framework and help understanding why and how calcification in coccolithophores will be affected by future climatic changes ${ }^{4,39}$, which in turn is key for developing predictive models of the future of calcification and the corresponding impact on climate.

\section{Methods}

Algal cultivation and induction of coccolith formation. Emiliania huxleyi strain AWI1516 (Alfred Wegner Institute), which produces coccoliths, was grown in artificial seawater medium Aquil, prepared according to the recipe of the National Center for Marine Algae and Microbiota, at $18{ }^{\circ} \mathrm{C}$ and a $12 / 12-\mathrm{h}$ light/dark cycle. The concentration of nitrate in the medium was $0.2 \mathrm{mM}$ and of phosphate $10 \mu \mathrm{M}$. The standard medium contained $10 \mathrm{mM} \mathrm{CaCl}_{2}$. Cells devoid of extracellular coccoliths were obtained by adding $1 / 50$ volume of $0.5 \mathrm{M}$ EDTA pH 8.0 to cultures and subsequent washing with fresh medium. This treatment dissolved extracellular calcite, but the calcite of coccoliths in statu nascendi (intracellular) remained undissolved. Cultures virtually free of coccolith calcite were obtained by repeated cultivation of EDTA-decalcified cells in modified artificial seawater medium, which contained $100 \mu \mathrm{M} \mathrm{CaCl}_{2}$. At $100 \mu \mathrm{M} \mathrm{Ca}^{2+}$, cells continued to divide, whereas coccolith formation was ceased (Supplementary Fig. 1). Calcite induction experiments were performed on calcite-free, logarithmic phase cells, $2 \mathrm{~h}$ after the start of the light phase. Coccolith formation was induced by the addition of $\mathrm{CaCl}_{2}$ to a final concentration of $10 \mathrm{mM}$. Algal cell density was measured using a Beckman Coulter Z2 particle counter.

Light microscopy. Light microscopy was performed on an Olympus BX-51 light microscope equipped with differential interference contrast and cross-polarization optics. Confocal fluorescence microscopy was performed using an inverted laser scanning microscope (SP5, Leica, Germany) equipped with a $\times 63$ water immersion objective (HCX PL APO CS, $\times 63.0$, numerical aperture 1.20 , Leica). Calcein-AM and chlorophyll were excited using the 488-nm line of an argon ion laser and fluorescence was recorded by photomultiplier detectors between 500 and $550 \mathrm{~nm}$, and 600 and $650 \mathrm{~nm}$, respectively. DAPI was imaged by two-photon excitation provided by a pulsed Ti:S laser tuned at $740 \mathrm{~nm}$. The fluorescence from DAPI was recorded by photomultiplier detectors between $420-450 \mathrm{~nm}$ and $500-550 \mathrm{~nm}$. Emission in the window $500-550 \mathrm{~nm}$ has been associated with DAPI-polyphosphate complexes ${ }^{27}$. Staining with DAPI and calcein-AM was achieved by incubating the cultures overnight with 7 and $5 \mu \mathrm{M}$, respectively.

Synchrotron-based XANES. The ACC standard was prepared by adding 1/25 volume of $1 \mathrm{M} \mathrm{CaCl}_{2}$ solution to $40 \mathrm{mM} \mathrm{Na}_{2} \mathrm{CO}_{3}$ solution at room temperature. The precipitates were collected by fast vacuum filtering, dehydrated and washed with ethanol, and stored in a vacuum desiccator. Calcite was prepared by adding $1 / 50$ volume of $5 \mathrm{M} \mathrm{CaCl}_{2}$ solution to $100 \mathrm{mM} \mathrm{Na}_{2} \mathrm{CO}_{3}$ solution. The solution was stirred for $24 \mathrm{~h}$ at room temperature. Calcite crystals were collected by centrifugation at $3,000 \mathrm{~g}$ for $5 \mathrm{~min}$, washed three times with $50 \mathrm{mM} \mathrm{NH}_{4} \mathrm{HCO}_{3}$ solutions and lyophilized. Culture aliquots before and after induction of coccolith formation were collected by centrifugation for $5 \mathrm{~min}$ at $4{ }^{\circ} \mathrm{C}$. Cell pellets were washed twice with $0.7 \mathrm{M}$ sorbitol (second time containing $10 \%$ dimethylsulphoxide) to remove extracellular calcium. The supernatant was poured off and the pellet was resuspended in the remaining solution. Cell suspensions were loaded into the wells (diameter $4 \mathrm{~mm}$, depth $1 \mathrm{~mm}$ ) of custommade $\mathrm{Cu}$ holders, snap-frozen and stored in liquid $\mathrm{N}_{2}$. The XANES spectra of the Ca K-edge were acquired at the LUCIA beamline at the SOLEIL Synchrotron Light Source (Saint-Aubin, France) by scanning the X-ray beam energy from 4,000 to $4,080 \mathrm{eV}$ in $1.0 \mathrm{eV}$ steps before the pre-edge and $0.2 \mathrm{eV}$ steps after the pre-edge. All samples were measured under vacuum and at cryo-conditions. The synchrotron ring energy was $2.75 \mathrm{GeV}$ and the current was up to $400 \mathrm{~mA}$. The X-ray energy was selected by a double-crystal $\mathrm{Si}(111)$ monochromator, which was calibrated using $\mathrm{Ti}$ foil and setting the edge at $4,966 \mathrm{eV}$. The beam size on the sample was $\sim 1.5 \times 1.5 \mathrm{~mm}^{2}$. Spectra of algal samples and $\mathrm{CaCl}_{2}$ solutions were collected on snap-frozen material, whereas the spectra for ACC and calcite were collected from powdered samples mounted on sticky tape. To improve the signal-to-noise ratios, all spectra were based on an average of three to five scans. Baseline subtraction, normalization, and data processing and analysis were performed using the Athena software package ${ }^{40}$. The edge was taken as the maximum of the third peak of the first derivative of the XANES data. Normalization was performed using a linear pre-edge function between 25 and $13 \mathrm{eV}$ below $E_{\mathrm{o}}$ and a quadratic polynomial for the post-edge between 26 and $90 \mathrm{eV}$ above $E_{0}$. Linear combination fitting was performed on all cell spectra using three calcium standards, $10 \mathrm{mM} \mathrm{CaCl}_{2}, \mathrm{ACC}$ and coccolith-bearing cells, $\mathrm{C}$ cells, whose spectrum was identical to crystalline calcite. The $10 \mathrm{mM} \mathrm{CaCl}_{2}$ solution represented free calcium ions.

Cryo-FIB milling and SEM imaging (cryo-FIB-SEM). E. huxleyi cells were collected by centrifugation, high-pressure frozen in a Leica HPM 100 apparatus using type-B sample holders (Ted Pella Inc., USA) and stored in liquid $\mathrm{N}_{2}$. Mounting and sputter coating, and cryo-FIB-SEM imaging were performed as described previously ${ }^{23}$. At liquid $\mathrm{N}_{2}$ temperature, the samples were mounted on a cryo-sample holder and transferred to the Leica SCD500 cryo-sputter coater, using the VCT100 cryo-transfer shuttle system (Leica Microsystems, Austria). The samples were sputter coated with a 6 -nm platinum layer at $0.06 \mathrm{~nm} \mathrm{~s}^{-1}$ and then transferred to the Zeiss Auriga60 FIB SEM microscope (Carl Zeiss Microscopy $\mathrm{GmbH}$, Germany), using the VCT100 shuttle system. Inside the microscope, a viewing channel for SEM imaging was directly milled into the sample surface using the $16-\mathrm{nA}$ FIB current at $30 \mathrm{kV}$ acceleration voltage (16-nA FIB probe at $30 \mathrm{kV})$. 
This initial coarse cross-section was fine polished using the 2-nA FIB probe at $30 \mathrm{kV}$. In a fully automated process, data cubes of serial SEM images were acquired. In a serial manner, a thin slice of material was removed by FIB milling followed by SEM imaging of the freshly exposed block face. For FIB slicing, the 240-pA FIB probe at $30 \mathrm{kV}$ was used. After each milling step (step thickness $15 \mathrm{~nm}$ ), the specimen was imaged by SEM at $2.53 \mathrm{kV}$ acceleration voltage using the $10-\mu \mathrm{m}$ aperture. For each slice secondary electron and backscattered electron images were acquired simultaneously using the in-lens secondary electron and the EsB detector $($ EsB grid $1,500 \mathrm{~V})$. The image resolution was 2,048 $\times 1,536$ pixels, lateral image pixel size was $5 \mathrm{~nm}$ and the slice thickness was $15 \mathrm{~nm}$. Images were recorded using line averaging $(N=51)$ and a dwell time of $200 \mathrm{~ns}$. The cycle time for recording an individual image was $36.1 \mathrm{~s}$. The milling time for removing each slice was $13.4 \mathrm{~s}$. The image series used to reconstruct the cell shown in Fig. $2 \mathrm{~d}$ consisted of 200 individual slices, representing a volume of $10.2 \times 7.6 \times 3 \mu \mathrm{m}$. Throughout the preparation and data acquisition process, the temperature was never higher than $-150^{\circ} \mathrm{C}$. Image processing and segmentation of data cubes were performed as follows: secondary electron images were Fourier filtered to remove the vertical stripes arising from the water fall effect by FIB milling and aligned automatically with the 'Linear Stack Alignment with SIFT' plugin of ImageJ (http:// rsbweb.nih.gov/ij/index.html), allowing only for translations. The same translation applied to each image of the secondary electron stack was then applied to the corresponding image of the EsB stack. Segmentation of structures of interest was performed using Amira 3D (FEI, USA). Organelles were segmented manually from secondary electron image stacks using the lasso tool. The Ca-rich bodies were segmented automatically from EsB image stacks using the Magic Wand tool. Surfaces were generated from segmented structures and smoothed. The MaterialStatistic module was used to calculate volumes and average grey scale intensities (EsB image stack only) of segmented structures. The grey scale intensities in the EsB image stacks were used for the quantification of calcium within the Ca-rich bodies. The grey scale intensities were calibrated against the backscattered coefficient $(\eta)$ of materials with known composition (internal standards). The calibration line was used to estimate $\eta$ at positions of interest. From $\eta$, the concentration of calcium was retrieved. In detail, lipid bodies, artificial seawater medium and external coccoliths were used as internal standards. The effective backscattered coefficient for every internal standard, $\eta_{\text {eff, was }}$ estimated using the mixture rule: $\eta_{\text {eff }}=\sum \eta_{\mathrm{i}} \times c_{i}$, where $c_{i}$ is the weight fraction of the $i$ th atom and $\eta_{i}$ the corresponding atomic backscattered coefficient. $\eta_{i}$ was calculated for each atom using the empirical Heinrich's formula under the assumption that $\eta$ for low atomic weight elements $(Z<20)$ does not change significantly with the primary beam energy ${ }^{41}$. Mass weight fractions for every atom (that is, the $c_{i}$ coefficients) were calculated under the assumption that (1) lipid bodies are constituted of linear hydrocarbons (largely $\mathrm{CH}_{2}$ ), (2) the $c_{i}$ 's of the extracellular solution are known from the composition of the seawater medium, (3) external coccoliths are made of pure calcite. From the calibration line (EsB intensity versus $\eta_{\text {eff }}$ ), the effective backscattered coefficient for the Ca-rich body was derived and, assuming that the Ca-rich body is a concentrated solution of electrolytes with a Ca:P atomic ratio of 1:2 (Supplementary table 1), the mass fraction of calcium ions was calculated. From this, the molarity of calcium inside the Ca-rich bodies was approximated by $1 / \rho_{\mathrm{s}}=c_{\mathrm{w}} / \rho_{\mathrm{w}}+\sum c_{i} / \rho_{i}$, where $c_{\mathrm{w}}$ and $\rho_{\mathrm{w}}$ are the weight fractions and the density of water, respectively, and $c_{i}$ is the weight fractions of the electrolytes and $\rho_{i}$ their densities calculated from their atomic radii.

\section{Analytical scanning transmission electron microscopy. Cells were high-} pressure frozen with 1-hexadecane (Sigma-Aldrich) as cryoprotectant and freeze-substituted with $2 \%(\mathrm{w} / \mathrm{v})$ osmium tetroxide (Electron Microscopy Sciences) and $8 \%(\mathrm{v} / \mathrm{v}) 2,2$-dimethoxypropane (Sigma-Aldrich) in acetone for 5 days at $-85^{\circ} \mathrm{C}$. Samples were warmed-up to room temperature over 2 days and embedded in Spurr's resin over 4 days. Resulting resin blocks were sectioned to $\sim 130 \mathrm{~nm}$ using a Leica UC-6 microtome. Thin sections were collected in pre-cooled ethylene glycol and mounted on copper grids. Dark-field electron imaging, EELS and EDX spectroscopy were performed on a Tecnai G2 F20 X-Twin microscope equipped with a field-emission electron source (operated at $200 \mathrm{kV}$ ), a Fishione HAADF detector at $330 \mathrm{~mm}$ camera length (diffraction contrast $+\mathrm{Z}$ contrast) for image acquisition in the STEM mode, a Gatan Tridiem imaging filter for acquisition of energy-filtered images and an EDAX Genesis X-ray analyser.

\section{Soft X-ray cryo-tomography and spectromicroscopy. Cells were EDTA}

decalcified and allowed to resume coccolith formation in normal medium for $3 \mathrm{~h}$ before collection by centrifugation. Cells in 3- $\mu \mathrm{l}$ medium and $1 \mu \mathrm{l}$ of a 100 -nm latex bead solution (2.5\%, Sigma-Aldrich) were placed together on top of Quantifoil R 2/2 holey film copper transmission electron microscopy grids (Quantifoil, Germany). The beads served as fiducial markers for the tomographic reconstruction. The sample was placed in a humidified chamber, manually blotted and plunge-frozen in liquid ethane using a Cryoplunge 3 system (Gatan Inc., USA). The frozen samples were kept at cryogenic conditions throughout shipment, mounting and imaging. Hydroxyapatite was synthesized by mixing $0.1 \mathrm{M} \mathrm{CaCl}_{2}$ solution with $0.1 \mathrm{M} \mathrm{Na}_{2} \mathrm{HPO}_{4}$ solution in a similar protocol to the synthesis of the calcium carbonate samples for XANES analysis. X-ray imaging was performed at the MISTRAL beamline (ALBA Synchrotron, Barcelona, Spain) ${ }^{42,43}$. Initially, a tilt series at $520 \mathrm{eV}$ X-ray energy was collected to allow a 3D volume reconstruction of cells and their internal structures. At this energy, carbon-rich, as well as Ca-rich, molecules have an intense contrast against the water-rich cytoplasm. The tilt series consisted of 121-131 images taken at $1^{\circ}$ degree intervals. Exposure time was $1-2 \mathrm{~s}$ to maximize signal-to-noise level and minimize radiation damage. The data sets were acquired using a zone plate objective lens with an outermost zone width of $\Delta r_{\mathrm{n}}=40 \mathrm{~nm}$. The effective pixel size in the images was $11.8 \mathrm{~nm}$. The projection images were normalized using flat-field images of 1-s exposure (incoming flux delivered by the capillary condenser lens) and corrected for changes in the electron beam current. Alignment and reconstruction of the tilt series were carried out with $\mathrm{IMOD}^{44}$. For tomogram reconstruction, the simultaneous iterative reconstruction technique ${ }^{45}$ was used. The resolution of the tomograms was calculated by the fourier shell correlation $\left(\mathrm{FSC}_{\mathrm{e} / \mathrm{o}}\right.$ ) criterion ${ }^{46}$ using a threshold of 0.25 . The visualization and segmentation of the final volumes were carried out using the software Amira 3D (FEI, USA). Energy scan series around the $\mathrm{Ca}_{2,3}$-edge followed the tilt series. In an energy scan, the same field of view was repeatedly imaged under changing X-ray energies starting at $347.7 \mathrm{eV}$ and going up to $354.7 \mathrm{eV}$ in $0.15 \mathrm{eV}$ steps, which is approximately half the energy resolution of the beamline for the used slits configuration. The objective zone plate lens and the charge-coupled device detector positions were automatically adjusted to maintain focus and constant magnification. Intracellular coccoliths were used as internal standard for the calibration of the absolute value of the energy. The extraction of a XANES spectrum for a specific area in the image was carried out by plotting the averaged intensity of pixels in this area, as a function of the energy at which the image was taken. First, the images of the different energies were aligned. For the spectrum of intracellular coccoliths, all pixels being part of a coccolith were manually marked in four cells and the intensity value plotted corresponded to the averaged intensity of all pixels. Analogously, the spectrum of Ca-rich bodies and the cytoplasm were obtained. Calcium localization in the samples was carried out by subtracting an image taken at $342 \mathrm{eV}$ (before the Ca L-edge) from an image taken at $353.2 \mathrm{eV}$ (at the peak of the second white line of the Ca L-edge). The contrast in the difference image is dominated by areas containing substantial $\mathrm{Ca}$ content. To exclude a possible radiation damage that can affect the spectroscopic features of the $\mathrm{Ca}$ atoms, a second energy scan was conducted after the first one. The identical spectra in both the scans confirmed that the irradiation did not affect the $\mathrm{Ca}$ local structure. The combination of $\mathrm{Ca}$ absorption at a specific location and the 3D data on the geometry of the $\mathrm{Ca}$-rich bodies enabled the estimation of $\mathrm{Ca}$ concentration from the acquired data. The pixel intensities in the difference images, acquired using energy-resolved $\mathrm{X}$-ray microscopy, give a semi-quantitative estimation of the content of $\mathrm{Ca}$ atoms in the X-ray path through this location. To estimate the concentration from the amount of calcium present in the X-ray path, the length of the Ca-rich volume at the relevant orientation was measured in the $3 \mathrm{D}$ reconstructed data. Intracellular coccoliths were used as internal standards to calibrate the Ca concentration in calcite which is $27 \mathrm{M}$. On four cells, the Ca concentration of calcite was measured with $13.7 \%$ s.d.

\section{References}

1. Sikes, C. S., Roer, R. D. \& Wilbur, K. M. Photosynthesis and coccolith formation: Inorganic carbon sources and net inorganic reaction of deposition. Limnol. Oceanogr. 25, 248-261 (1980).

2. O'Dea, S. A. et al. Coccolithophore calcification response to past ocean acidification and climate change. Nat. Commun. 5, 5363 (2014).

3. Iglesias-Rodriguez, M. D. et al. Phytoplankton calcification in a high- $\mathrm{CO}_{2}$ world. Science 320, 336-340 (2008).

4. Beaufort, L. et al. Sensitivity of coccolithophores to carbonate chemistry and ocean acidification. Nature 476, 80-83 (2011).

5. Stoll, H. M. \& Ziveri, P. in Coccolithophores (eds Thierstein, H. R. \& Young, J. R.) Ch. 20, 529-562 (Springer, 2004).

6. Wilbur, K. M. \& Watabe, N. Experimental studies on calcification in molluscs and the alga Coccolithus huxleyi. Ann. NY Acad. Sci. 109, 82-112 (1963).

7. Young, J. R. \& Ziveri, P. Calculation of coccolith volume and it use in calibration of carbonate flux estimates. Deep Sea Res. Part II 47, 1679-1700 (2000).

8. Holtz, L. M., Thoms, S., Langer, G. \& Wolf-Gladrow, D. A. Substrate supply for calcite precipitation in Emiliania huxleyi: assessment of different model approaches. J. Phycol. 49, 417-426 (2013).

9. Gussone, N. et al. Cellular calcium pathways and isotope fractionation in Emiliania huxleyi. Geology 34, 625-628 (2006).

10. Klaveness, D. Coccolithus huxleyi (Lohmann) I. Morphological investigations on the vegetative cell and the process of coccolith formation. Protistologica 8 , 335-346 (1972).

11. van der Wal, P., de Jong, E. W., Westbroek, P., de Bruijn, W. C. \& Mulder-Stapel, A. A. Ultrastructural polysaccharide localization in calcifying and naked cells of the coccolithophorid Emiliania huxleyi. Protoplasma 118, 157-168 (1983).

12. Taylor, A. R., Russell, M. A., Harper, G. M., Collins, T. F. T. \& Brownlee, C. Dynamics of formation and secretion of heterococcoliths by Coccolithus pelagicus ssp. braarudii. Eur. J. Phycol. 42, 125-136 (2007). 
13. Seto, J. et al. Structure-property relationships of a biological mesocrystal in the adult sea urchin spine. Proc. Natl Acad. Sci. USA 109, 3699-3704 (2012).

14. Vidavsky, N. et al. Initial stages of calcium uptake and mineral deposition in sea urchin embryos. Proc. Natl Acad. Sci. USA 111, 39-44 (2014).

15. Baumgartner, J. et al. Magnetotactic bacteria form magnetite from a phosphaterich ferric hydroxide via nanometric ferric (oxyhydr)oxide intermediates. Proc. Natl Acad. Sci. USA 110, 14883-14888 (2013).

16. Politi, Y. et al. Structural characterization of the transient amorphous calcium carbonate precursor phase in sea urchin embryos. Adv. Funct. Mater. 16, 1289-1298 (2006).

17. Schneider, G. et al. Three-dimensional cellular ultrastructure resolved by X-ray microscopy. Nat. Methods 7, 985-987 (2010).

18. Kapishnikov, S. et al. Oriented nucleation of hemozoin at the digestive vacuole membrane in Plasmodium falciparum. Proc. Natl Acad. Sci. USA 109, 11188-11193 (2012)

19. Gong, Y. U. T. et al. Phase transitions in biogenic amorphous calcium carbonate. Proc. Natl Acad. Sci. USA 109, 6088-6093 (2012).

20. Beniash, E., Metzler, R. A., Lam, R. S. K. \& Gilbert, P. U. P. A. Transient amorphous calcium phosphate in forming enamel. J. Struct. Biol. 166, 133-143 (2009).

21. DeVol, R. T. et al. Oxygen spectroscopy and polarization-dependent imaging contrast (PIC)-mapping of calcium carbonate minerals and biominerals. J. Phys. Chem. B 118, 8449-8457 (2014)

22. DeVol, R. T. et al. Nanoscale transforming mineral phases in fresh nacre. J. Am. Chem. Soc. 137, 13325-13333 (2015).

23. Schertel, A. et al. Cryo FIB-SEM: volume imaging of cellular ultrastructure in native frozen specimens. J. Struct. Biol. 184, 355-360 (2013).

24. Wu, J. S. et al. Imaging and elemental mapping of biological specimens with a dual-EDS dedicated scanning transmission electron microscope. Ultramicroscopy 128, 24-31 (2013).

25. van der Wal, P., Leunissen-Bijvelt, J. J. M. \& Verkleij, A. J. Ultrastructure of the membranous layers enveloping the cell of the coccolithophorid Emiliania huxleyi. J. Ultrastruct. Res. 91, 24-29 (1985).

26. Lawrence, J. R. et al. Scanning transmission X-ray, laser scanning, and transmission electron microscopy mapping of the exopolymeric matrix of microbial biofilms. Appl. Environ. Microb. 69, 5543-5554 (2003).

27. Omelon, S. J. \& Grynpas, M. D. Relationships between polyphosphate chemistry, biochemistry and apatite biomineralization. Chem. Rev. 108, 4694-4715 (2008).

28. Van Der Veer, J. \& Leewis, R. J. Pavlova ennorea sp. nov., a Haptophycean alga with a dominant palmelloid phase, from England. Acta Bot. Neerlandica 26, 159-176 (1977).

29. Read, B. A. et al. Pan genome of the phytoplankton Emiliania underpins its global distribution. Nature 499, 209-213 (2013).

30. Gomes, F. M. et al. New insights into the in situ microscopic visualization and quantification of inorganic polyphosphate stores by 4',6-diamidino-2phenylindole (DAPI)-staining. Eur. J. Histochem. 57, e34 (2013).

31. Bentov, S., Brownlee, C. \& Erez, J. The role of seawater endocytosis in the biomineralization process in calcareous foraminifera. Proc. Natl Acad. Sci. USA 106, 21500-21504 (2009).

32. Tambutte, E. et al. Calcein labelling and electrophysiology: insights on coral tissue permeability and calcification. Proc. Biol. Sci. 279, 19-27 (2012).

33. Marsh, M. E. in Biomineralization: From Biology to Biotechnology and Medical Application (ed. Baeuerlein, E.) (Wiley-VCH, 2000).

34. Taylor, M., Simkiss, K. \& Greaves, G. N. Amorphous structure of intracellular mineral granules. Biochem. Soc. Trans. 14, 549-552 (1986).

35. Ziegler, A., Fabritius, H. \& Hagedorn, M. Microscopical and functional aspects of calcium-transport and deposition in terrestrial isopods. Micron 36, 137-153 (2005).

36. Docampo, R., de Souza, W., Miranda, K., Rohloff, P. \& Moreno, S. N. J. Acidocalcisomes-conserved from bacteria to man. Nat. Rev. Microbiol. 3, 251-261 (2005)

37. Lessard, E. J., Merico, A. \& Tyrrell, T. Nitrate: phosphate ratios and Emiliania huxleyi blooms. Limnol. Oceanogr. 50, 1020-1024 (2005).

38. Marsh, M. E. Polyanion-mediated mineralization-a kinetic analysis of the calcium-carrier hypothesis in the phytoflagellate Pleurochrysis carterae. Protoplasma 190, 181-188 (1996).

39. Lohbeck, K. T., Riebesell, U. \& Reusch, T. B. H. Adaptive evolution of a key phytoplankton species to ocean acidification. Nat. Geosci. 5, 346-351 (2012).
40. Ravel, B. \& Newville, M. ATHENA, ARTEMIS, HEPHAESTUS: Data analysis for X-ray absorption spectroscopy using IFEFFIT. J. Synchroton Radiat. 12, 537-541 (2005).

41. Reimer, L. Image Formation in Low-Voltage Scanning Electron Microscopy (SPIE Optical Engineering Press, 1993).

42. Pereiro, E., Nicolás, J., Ferrer, S. \& Howells, M. R. A soft X-ray beamline for transmission X-ray microscopy at ALBA. J. Synchrotron Radiat. 16, 505-512 (2009).

43. Sorrentino, A. et al. MISTRAL: a transmission soft X-ray microscopy beamline for cryo nano-tomography of biological samples and magnetic domains imaging. J. Synchrotron Radiat 22, 1112-1117 (2015).

44. Kremer, J. R., Mastronarde, D. N. \& McIntosh, J. R. Computer visualization of three-dimensional image data using IMOD. J. Struct. Biol. 116, 71-76 (1996).

45. Gilbert, P. Iterative methods for the three-dimensional reconstruction of an object from projections. J. Theor. Biol. 36, 105-117 (1972).

46. Cardone, G., Grünewald, K. \& Steven, A. C. A resolution criterion for electron tomography based on cross-validation. J. Struct. Biol. 151, 117-129 (2005).

47. Eichert, D., Salomé, M., Banu, M., Susini, J. \& Rey, C. Preliminary characterization of calcium chemical environment in apatitic and non-apatitic calcium phosphates of biological interest by X-ray absorption spectroscopy. Spectrochim. Acta B 60, 850-858 (2005).

\section{Acknowledgements}

We thank Wouter Habraken and Zhaoyong Zou for the synthesis of ACC and Jens Baumgartner (all Max-Planck Institute of Colloids and Interfaces, Potsdam) for help with XANES measurements. We are grateful to Ralph Bock (Max-Planck Institute of Molecular Plant Physiology, Potsdam) and Nils Kröger (TU Dresden, Dresden) for critically reading the manuscript. We acknowledge SOLEIL for provision of synchrotron radiation facilities and we would like to thank Nicolas Trcera and Benedikt Lassalle (both SOLEIL synchrotron, Gif-sur-Yvette) for assistance in using the LUCIA beamline. X-ray microscopy was conducted at the MISTRAL beamline at ALBA Synchrotron, Barcelona, and received funding from the European Community's Seventh Framework Programme (FP7/2007-2013) under BioStruct-X (grant agreement N283570). This research was supported by the Max-Planck Society, Deutsche Forschungsgemeinschaft (DFG) grants Sche1637/3-1 and Sche1637/4-1 to André Scheffel, and FA835/9-1 to D.F. A.G. is supported by an Alexander von Humboldt postdoctoral fellowship. P.K. was supported by the European Union Seventh Framework Programme (FP7 2007-2013) under the WallTraC project (Grant Agreement number 263916). D.F. acknowledges financial support from the European Research Council (starting grant MB2 N256915).

\section{Author contributions}

S.S., M.A.H., A.G., L.B., Y.P., D.F. and André Scheffel designed the research; L.B., S.S., Y.P., and Andreas Schertel contributed cryo-FIB-SEM data; A.G., Andrea Sorrentino and E.P. contributed X-ray microscopy data; S.S. and André Scheffel developed protocols for inducing coccolith formation; S.S., M.A.H., Y.P. and André Scheffel contributed XANES data; S.S., M.A.H., A.G., P.K. and R.W. contributed data on thin-sectioned cells; S.S. and M.B. contributed confocal images; S.S., M.A.H., A.G., L.B., Y.P., D.F. and André Scheffel wrote the paper.

\section{Additional information}

Supplementary Information accompanies this paper at http://www.nature.com/ naturecommunication

Competing financial interests: The authors declare no competing financial interests

Reprints and permission information is available online at http://npg.nature.com/ reprintsandpermissions/

How to cite this article: Sviben, S. et al. A vacuole-like compartment concentrates a disordered calcium phase in a key coccolithophorid alga. Nat. Commun. 7:11228 doi: $10.1038 /$ ncomms11228 (2016)

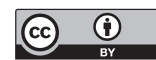

This work is licensed under a Creative Commons Attribution 4.0 International License. The images or other third party material in this article are included in the article's Creative Commons license, unless indicated otherwise in the credit line; if the material is not included under the Creative Commons license, users will need to obtain permission from the license holder to reproduce the material. To view a copy of this license, visit http://creativecommons.org/licenses/by/4.0/ 